Mamata Patil, Mahesha, and C.S.K. Raju*

\title{
Convective conditions and dissipation on Tangent Hyperbolic fluid over a chemically heating exponentially porous sheet
}

https://doi.org/10.1515/nleng-2018-0003

Received January 5, 2018; revised June 1, 2018; accepted August 3, 2018.

\begin{abstract}
In this present analysis we investigated the steady-state magnetohydrodynamic boundary layer flow of tangent hyperbolic fluid over an exponentially stretching surface in the presence of heat source and chemical reaction. The chemical reaction with combination of exponential surface has significance in many industrial and manufacturing systems. The partial nonlinear differential equations are transformed into ordinary differential equations by using the similarity conversion and the accomplished boundary layer ordinary differential equations are elucidated numerically by using Shooting technique. The effects of numerous non-dimensional governing factors on velocity, temperature and concentration profiles were depicted graphically and analyzed in detail. The numerically computed results of Skin friction factor, Nusselt and Sherwood numbers are presented in tabular form for suction and injection cases separately. Heat transfer rate at the surface increases with increasing values of power law of index and whereas it declines with the magnetic field, heat source and chemical reaction parameters. It observed that Biot number enhances the skin friction, Nusselt number and decrease the Sherwood number. Heat transfer rate and mass transfer rate increases and skin friction decreases with increasing Eckert number.
\end{abstract}

Keywords: Non-Newtonian tangent hyperbolic fluid; viscous dissipation; Convective conditions; Heat source; Chemical reaction; exponentially sheet

Mamata Patil, Department of Mathematics, University B.D.T College of Engineering, Davangere, Karnataka -57700 , India, E-mail: mamatapatil72@gmail.com

Mahesha, Department of Mathematics, University B.D.T College of Engineering, Davangere, Karnataka -57700, India, E-mail:

maheshubdt@gmail.com

*Corresponding Author: C.S.K. Raju, Department of Mathematics, GITAM School of Technology, Bangalore, India, E-mail: sivaphd90@gmail.com

\section{Introduction}

A non-Newtonian fluid is a fluid that does not follow Newton's Law of Viscosity. Most commonly, the viscosity of non-Newtonian fluids is dependent on shear rate or shear rate history. Some non-Newtonian fluids with shear-independent viscosity, however, still exhibit normal stress-differences or other non-Newtonian behavior. Many salt solutions and molten polymers are nonNewtonian fluids, as are many commonly found substances such as ketchup, custard, toothpaste, starch suspensions, paint, blood and shampoo. In a Newtonian fluid, the relation between the shear stress and the shear rate is linear, passing through the origin, the constant of proportionality being the coefficient of viscosity. In a nonNewtonian fluid, the relation between the shear stress and the shear rate is different. The fluid can even exhibit timedependent viscosity. Therefore, a constant coefficient of viscosity cannot be defined. Nadeem and Akram [1] investigated the MHD peristaltic flow of a hyperbolic tangent fluid model in a vertical asymmetric channel under the assumption of long wavelength approximation. The nonlinear boundary layer flow and heat transfer of an incompressible non-Newtonian tangent hyperbolic fluid from a sphere using finite difference Keller box method examined by Abdul et al. [2]. Gnaneswara Reddy et al. [3] emphases on the magneto hydrodynamics flow of non-Newtonian tangent hyperbolic fluid towards a cylinder with thermal radiation. The laminar flow and heat transfer of powerlaw non-Newtonian fluids over a stretching sheet with the sheet velocity distribution discussed by $\mathrm{Xu}$ and Liao [4]. Megahed [5] considered for the special effects of heat flux and thermal buoyancy on the two-dimensional flow and heat transfer of a non-Newtonian power-law fluid over a non-linearly vertical surface in the incidence of thermal radiation. The unsteady magneto hydrodynamic flow and heat transfer of a fluid over a stretching sheet in the existence of viscous dissipation and heat source investigated by Gnaneswara reddy et al. [6]. Choudhray et al. [7] designate an unsteady flow of a viscous incompressible electri- 
cally conducting fluid over a stretching permeable surface in suction or injection cases.

The tangent hyperbolic fluid model is sufficient competent to define shear thinning phenomenon. It measures the fluid nourishing less flow with additional rate of shear stress. In literature many considered were reported on tangent hyperbolic fluid by seeing various physical aspects. The convective heat transfer analysis of non-Newtonian tangent hyperbolic fluid in a certain way by enchanting the effects of viscous dissipation over a non-linear stretching sheet by considering HAM and shooting method examined by Hussain et al. [8]. Partha et al. [9] studied the mixed convection flow and heat transfer from an exponentially stretching surface in presence of viscous dissipation. The two dimensional flow of heat and mass transfer of MHD boundary layer flow of tangent hyperbolic fluid in the existence of thermal dispersion. The electrically conducting tangent hyperbolic fluid is a four constant fluid model accomplished of describing shear thinning effects investigated by Uma and Koteswara [10]. Mamatha et al. [11] considered the incompressible MHD Carreau Dusty fluid over a stretching sheet with exponentially moldering heat source. The non-isothermal steady-state boundary layer flow and heat transfer of tangent hyperbolic nonNewtonian fluid from a vertical permeable cone with magnetic field are considered by Gaffar et al. [12]. Raju et al. [13] evaluated the flow, heat and mass transfer behavior of MHD Casson fluid past an exponentially stretching surface, offered dual solutions by comparing the results of the Casson fluid with the Newtonian fluid. The magneto hydrodynamics boundary layer flow and heat transfer of a fluid with viscosity through porosity towards a stretching sheet by compelling in to the effects of viscous dissipation in incidence of heat source/sink is deliberated by Dessie and Kishan [14]. Naseer et al. [15] studied the boundary layer flow and heat transfer of a non-Newtonian hyperbolic tangent fluid flowing over a vertical exponentially stretching cylinder.

The concept of viscosity is commonly used in fluid mechanics to characterize the shear properties of a fluid; it can be inadequate to describe non-Newtonian fluids. They are best studied through several other rheological properties that relate stress and strain rate tensors under many different flow conditions such as oscillatory shear or extensional flow which are measured using different devices or rheometers. The properties are better studied using tensor-valued constitutive equations, which are common in the field of continuum mechanics. Abel et al. [16] considered the flow of a power-law fluid due to a linear stretching sheet and heat transfer appearances using variable thermal conductivity is calculated in the presence of a non-uniform heat source/sink. The peristaltic transport of a hyperbolic tangent fluid through a porous medium in a symmetric inclined channel under the assumptions of low Reynolds number and long wavelength examined by Jyothi et al. [17]. Vendabai [18] considered the unsteady boundary layer flow of a nanofluid over a stretching surface with variable radiation effect in the incidence of heat source. The heat transfer features of an unsteady flow of a nanofluid past a stretching sheet with a convective boundary condition are deliberate by Mansur and Ishak [19]. The effect of transverse magnetic field and heat source on the boundary layer flow of a Casson nanofluid over a cylinder stretching exponentially studied by Sarojamma and Vendabai [20]. Loganathan et al. [21] considered the properties of thermal radiation and magnetic field on unsteady convective flow of a nanofluid past an impetuously started infinite vertical plate in existence of heat source by using Laplace Transform method. Ganesh Kumar et al. [22] investigated the statistical study on squeezed flow of tangent hyperbolic fluid over a sensor surface with thermal conductivity elucidated numerically by RKF45 technique. The impact of heat absorption on tangent hyperbolic Nano fluid neighboring the stagnation point over cylinder and established the model of tangent hyperbolic Nano fluid with Brownian motion and thermophoresis effects scrutinized by Salahuddin et al. [23]. Brinkman [24] originally included the viscous dissipation effects into account; he analyzed the properties of viscous dissipation on capillary flows. Ramachandra Prasad et al. [25] observed the nonlinear free convection boundary layer flow and heat transfer of Tangent Hyperbolic fluid from a vertical porous plate with velocity slip and thermal jump effects. The Dufour effect is the energy flux due to a mass concentration gradient occurring as a coupled effect of irreversible processes. It is the reciprocal phenomenon to the Soret effect. The concentration gradient results in a temperature change. For binary liquid mixtures, the Dufour effect is usually considered negligible, whereas in binary gas mixtures the effect can be significant. The effects of chemical reaction on mixed convection flow along a sphere in non-Darcian porous media. Intention the sphere surface is conserved at uniform temperature and species concentration for both cases of heated and cooled sphere deliberated by [26-29]. Chamkha and Rashad [30] investigated on the Soret and Dufour effects on unsteady coupled heat and mass transfer by mixed convection flow over a vertical cone rotating in an ambient fluid with a time-dependent angular velocity in the incidence of a magnetic field and chemical reaction. The unsteady and coupled heat and mass transfer by MHD mixed convective boundary-layer flow of an electrically conducting fluid over an impulsively stretched ver- 
tical surface in an unbounded inert fluid with aiding external flow in the presence of a transverse magnetic field, chemical reaction, and Soret and Dufour effects described by [31-33]. Rashad and Chamkha [34] studied the MHD free convective heat and mass transfer of a viscous, electrically conducting and chemically-reacting fluid contiguous to a vertical stretching sheet embedded in a saturated porous medium.

The objective of this present study we analyzed the flow, heat and mass transfer behavior of tangent hyperbolic fluid over an exponentially stretching surface in the occurrence of heat source. The partial nonlinear differential equations are transformed into ordinary differential equations by using the similarity conversion and the accomplished boundary layer ordinary differential equations are elucidated numerically by using Shooting technique. The effects of numerous non-dimensional governing factors on velocity, temperature and gas concentration profiles are debated and presented graphically. Heat transfer rate at the surface increases with increasing values of power law of index and whereas it decline with magnetic field parameter, heat source parameter, chemical reaction parameter. Also, the Skin friction factor, Nusselt and Sherwood numbers are analyzed and presented in tabular form for permeable sheet and injection case of permeable sheet separately.

\section{Mathematical formulation}

We deliberate the steady, incompressible, Magneto hydrodynamic tangent fluid over a nonlinearly exponentially stretching sheet which overlaps with the horizontal $y=0$. The fluid flow is narrowed to $y>0$. We applied an inconstant magnetic field and there is no applied power, which indicates the absence of an electrical field. Also it is considered that the induced magnetic field is lesser compared to the exterior magnetic field. This indicates that a trivial Reynolds number exoduses in this study. Along with this we deliberated heat source and chemical reaction to the flow. Two equivalent and opposite forces are applied along the $x$ axis, so that the wall is stressed keeping the origin fixed. The rheological expression of state for an extra stress tensor $S$ of tangent hyperbolic fluid is as follows.

$$
S=\left\{\mu_{\infty}+\left(\mu_{0}+\mu_{\infty}\right) \tanh (\overline{\bar{y}})^{n}\right\} A
$$

Where $\mu_{\infty}$ the infinite shear rate viscosity, $\mu_{0}$ zero shear rate viscosity, is the time dependent constant, $n$ power law of index and $\bar{y}$ is given by,

$$
\bar{y}=\sqrt{1 / 2 \operatorname{tr}\left(A_{1}^{2}\right)}
$$

Assuming that $\mu_{\infty}=0$ is assimilated in equation (1), also as tangent hyperbolic fluid designates the shear thinning spectacles, thus $\bar{i} y<1$. After using these abovementioned hypotheses in equation (1); it takes the form as follows.

$$
S=\mu_{0}\left[(\overline{\bar{x} y})^{n}\right] A_{1}
$$

After universalization the above equation convert to:

$$
S=\mu_{0}\left[1+n(\overline{\bar{\tau} y}-1)^{n}\right] A_{1}
$$

The leading equations in components form after inferring boundary layer the basic continuity, momentum and energy equations (Partha et al. (2005)), Raju et al (2016) and (Uma and Koteswara (2017)) are given by.

$$
\begin{gathered}
\frac{\partial u}{\partial x}+\frac{\partial v}{\partial y}=0 \\
u \frac{\partial u}{\partial x}+v \frac{\partial u}{\partial y}=v(1-n) \frac{\partial^{2} u}{\partial y^{2}}+\sqrt{2} v n \tau\left(\frac{\partial u}{\partial y}\right)\left(\frac{\partial^{2} u}{\partial y^{2}}\right) \\
-\frac{\sigma B^{2}}{\rho} u+g \beta_{T}\left(T-T_{\infty}\right)+g \beta_{C}\left(C-C_{\infty}\right)-\frac{v}{K_{0}} u \\
u \frac{\partial T}{\partial x}+v \frac{\partial T}{\partial y}=\frac{k}{\rho C_{p}} \frac{\partial^{2} T}{\partial y^{2}}-\frac{1}{\rho C_{p}} \frac{\partial q_{r}}{\partial y}+\frac{v}{C_{p}}(1-n)\left(\frac{\partial u}{\partial y}\right)^{2} \\
+\frac{v n \tau}{C_{p} \sqrt{2}}\left(\frac{\partial u}{\partial y}\right)\left(\frac{\partial u}{\partial y}\right)^{2}-Q_{0}\left(T-T_{\infty}\right) \\
u \frac{\partial C}{\partial x}+v \frac{\partial C}{\partial y}=D_{m} \frac{\partial^{2} C}{\partial y^{2}}-K_{l}\left(C-C_{\infty}\right)
\end{gathered}
$$

Subject to the boundary conditions;

$$
\begin{gathered}
u=U_{0} e^{N x / 2 L}, v=v_{0} e^{N x / 2 L},-k_{f} \frac{\partial T}{\partial y}=h_{f}\left(T-T_{\infty}\right), C=C_{w} \\
\text { at; } y=0, u \rightarrow 0, T \rightarrow T_{\infty}, C \rightarrow C_{\infty} a s ; y \rightarrow \infty
\end{gathered}
$$

Where $u$ and $v$ the velocity components in the $\mathrm{x}$ and $\mathrm{y}$, and $U$ are is the constant velocity, $v$ is the kinematic viscosity, $\rho$ is the fluid density, $g$ is the acceleration due to gravity, $T$ is the fluid temperature, $\sigma$ is electric conductivity, $B=B_{0} e^{N x / 2 L}$ is the variable magnetic field, $k$ is the thermal conductivity of the fluid, $\rho C_{p}$ is the heat capacitance of fluid, $\beta_{T}$ is the thermal expansion coefficient, $\beta_{C}$ is the concentration expansion coefficient, $Q_{0}=Q e^{N x / L}$ is the variable heat source parameter, $q_{r}$ the radiative heat flux, $D_{m}$ is the coefficient of the mass diffusivity, $C$ is the 
concentration of the fluid, $K_{l}=k_{0} e^{N x / L}$ is the chemical reaction parameter and $N$ is the exponential parameter.

The radiative heat flux $q_{r}$ under Roseland approximation is expressed as

$$
q_{r}=\frac{-4 \sigma^{\star}}{3 k^{\star}} \frac{\partial T^{4}}{\partial y}
$$

Where $k^{\star}$ is the mean absorption coefficient and $\sigma^{\star}$ is the Stefan-Boltzmann constant, the above radiative heat flux $q_{r}$ is effective at a point away from boundary layer surface in an intensive absorption flow. Considering that the temperature difference within the flow is sufficiently small then $T^{4}$ may be expressed as a linear function of temperature $T$. Expanding $T^{4}$ by Taylor's series about temperature $T_{\infty}$ and which after neglecting higher order terms takes the forms,

$$
T^{4} \cong 4 T_{\infty}^{3} T-3 T_{\infty}^{4}
$$

Using equation (10) and (11) in equation (7) is reduced to:

$$
\begin{aligned}
& u \frac{\partial T}{\partial x}+v \frac{\partial T}{\partial y}=\frac{k}{\rho C_{p}} \frac{\partial^{2} T}{\partial y^{2}}+\frac{16 \sigma^{\star} T_{\infty}^{3}}{3 \rho C_{p} k^{\star}} \frac{\partial^{2} T}{\partial y^{2}}+\frac{v}{C_{p}}(1-n)\left(\frac{\partial u}{\partial y}\right)^{2} \\
& +\frac{v n \tau}{C_{p} \sqrt{2}}\left(\frac{\partial u}{\partial y}\right)\left(\frac{\partial u}{\partial y}\right)^{2}
\end{aligned}
$$

To find consistent set of nonlinear ordinary differential equations, use the below defined similarity transformations;

$\varsigma=\sqrt{U_{0} / 2 v L} e^{N x / 2 L} y, v=-\sqrt{v U_{0} / 2 L} e^{Z x / 2 L} N\left\{f(\varsigma)+\varsigma f^{\prime}(\varsigma)\right\}$

$$
u=U_{0} e^{N x / 2 L} f^{\prime}(\varsigma), T=T_{\infty}+T_{0} e^{2 N x / L}, C=C_{\infty}+C_{0} e^{2 N x / L}
$$

Where $T_{\infty}$ is the ambient fluid temperature, $C_{\infty}$ is the ambient fluid concentration, $L$ is the characteristic length, $U_{0}$ is the fluid velocity.

In view of Equations (9) to (13) in equations (6), (8) and (12) reduced to:

$$
\begin{aligned}
& (1-n) f^{\prime \prime \prime}+N\left\{f f^{\prime \prime}-2\left(f^{\prime}\right)^{2}\right\}+n W e f^{\prime \prime \prime} f^{\prime \prime}+2 G r \theta \\
& +2 G c \phi-(M+K) f^{\prime}=0 \\
& \frac{1}{\operatorname{Pr}}\left(\frac{4}{3} R+1\right) \theta^{\prime \prime}+N\left\{4 f^{\prime} \theta-f \theta^{\prime}\right\}+(1-n)\left(f^{\prime \prime}\right)^{2} \\
& +\frac{n W e E c}{2}\left(f^{\prime \prime}\right)^{3}-Q_{H} \theta=0 \\
& \phi^{\prime \prime}+N S c\left\{4 f^{\prime} \phi-f \phi^{\prime}\right\}-S c K_{l} \phi=0
\end{aligned}
$$

The transformed boundary conditions are:

$f(\xi)=f_{w}, f^{\prime}(\xi)=1, \theta^{\prime}(\xi)=-B i[1-\theta(\xi)], \phi(\xi)=1 a t ; \xi=1$ $f^{\prime}(\xi) \rightarrow 0, \theta(\xi) \rightarrow 0, \phi(\xi) \rightarrow 0$ at $; \xi \rightarrow \infty$
Where primes denote differentiation with respect to the similarity variable $\xi, f_{w}=v_{0} / \sqrt{U_{0} v / 2 L} f_{w}$ is the suction or permeable for $f_{w}>0, f_{w}=0$ is for impermeable surface and $f_{w}<0$ for injection.

Where $B i=h_{f} / k_{f} \sqrt{2 v L / U_{0}}$, We $=$ $\tau / \sqrt{v L}\left(U_{0} e^{N x / L}\right)^{3 / 2}, \operatorname{Pr}=v / \alpha, M=2 \sigma B_{0}^{2} L / r h o U_{0}$, $K=\rho U_{0} / K_{0}, Q_{H}=Q_{0} L / T_{0} U_{0}, E c=U_{0}^{2} / L c_{p} T_{0}$, $S c=v / D_{m}, K_{l}=k_{0} L / C_{0} U_{0}, G r=g \beta_{T} L T_{0} / U_{0}^{2}$, $G c=g \beta_{c} L C_{0} / U_{0}^{2}$.

The quantities of practical interest in this study are the skin friction coefficient $(\mathrm{Cf})$, local Nusselt number $(\mathrm{Nu})$ and Sherwood number $(S h)$ which are defined as

$$
\begin{aligned}
& \operatorname{CfRe}_{x}^{1 / 2}=((1-n)+n W e) f^{\prime \prime}(0), \\
& \operatorname{NuRe}_{x}^{-1 / 2}=-\left(1+\frac{4}{3} R\right) \theta^{\prime}(0), \\
& \operatorname{ShRe}_{x}^{-1 / 2}=-\phi^{\prime}(0)
\end{aligned}
$$

Where $R e_{x}=U x / v_{f}$ the local Reynolds number.

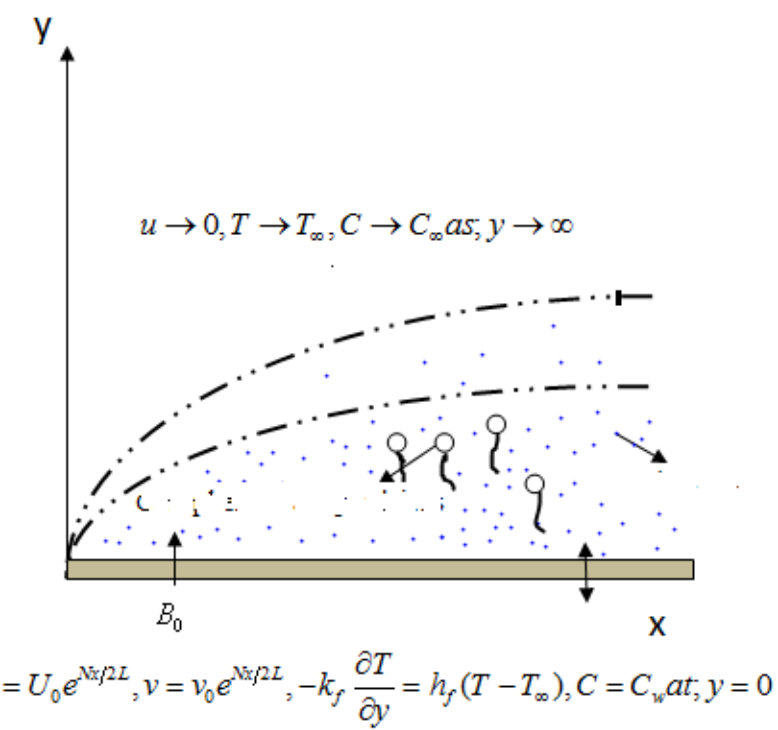

Fig. 1: Physical flow model.

\section{Results and Discussion}

The system of ordinary differential equations (14) to (16) with the boundary conditions (17) are elucidated numerically by using Shooting technique. The achieved results show the effects of the different non-dimensional governing parameters, namely Magnetic field parameter $(M)$, heat source parameter $\left(Q_{H}\right)$, Biot Number $(B i)$, Eckert 


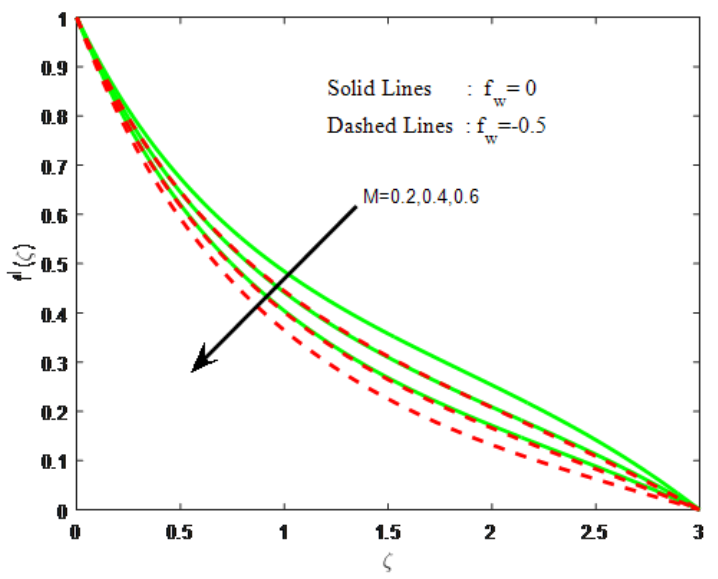

Fig. 2: The effect of Magnetic field $M$ on velocity field profile

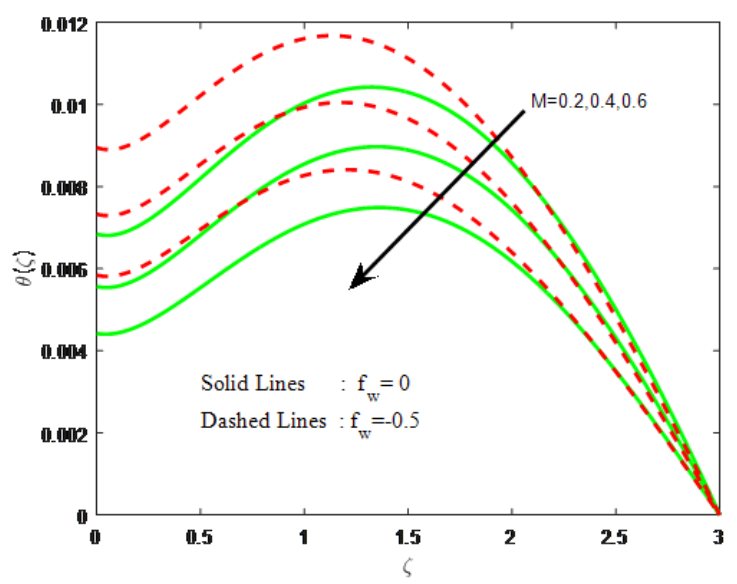

Fig. 3: The effect of Magnetic field $M$ on temperature field

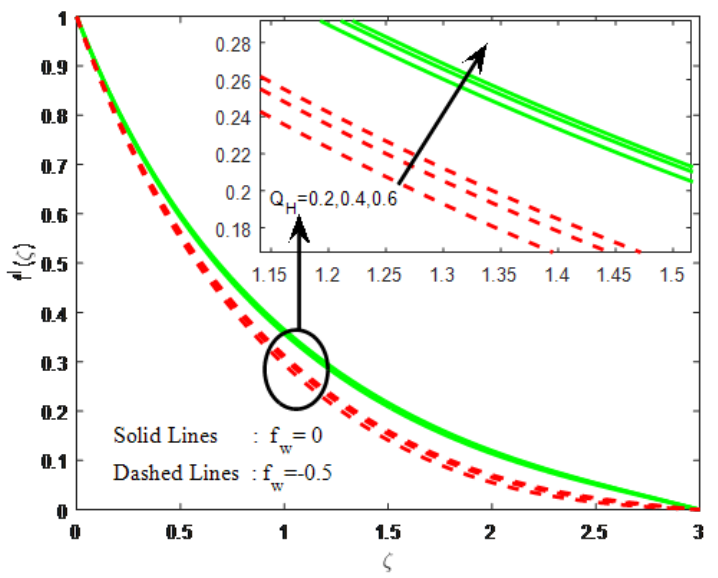

Fig. 4: The effect of heat source parameter $Q_{H}$ on velocity field

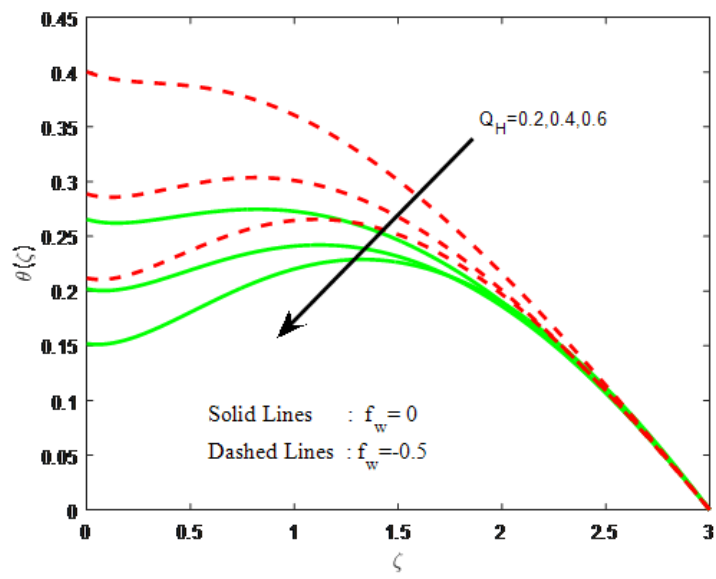

Fig. 5: The effect of heat source parameter $Q_{H}$ on temperature field

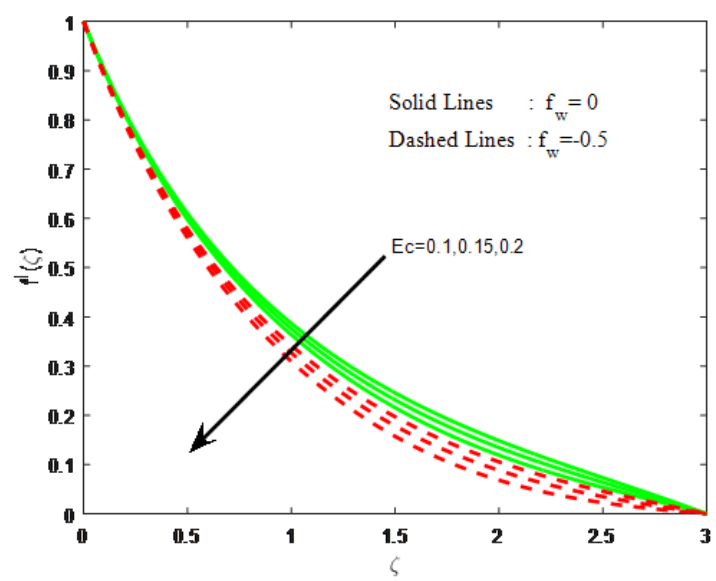

Fig. 6: The effect of Biot number Bi on velocity field

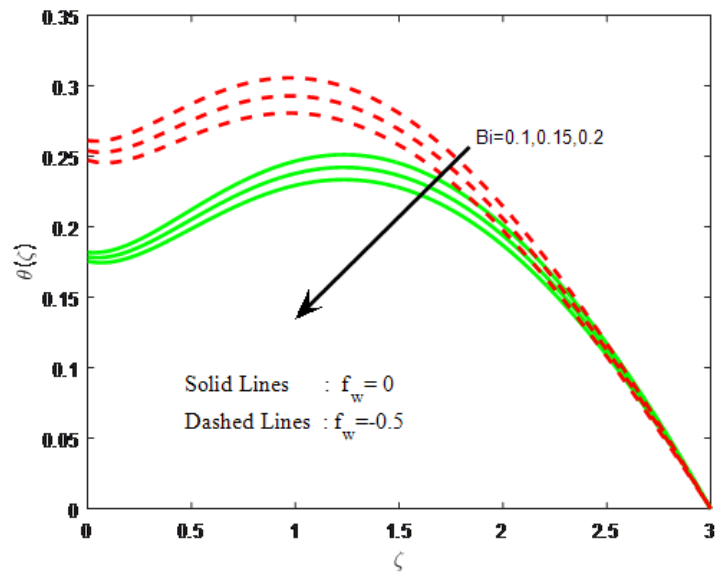

Fig. 7: The effect of Biot number $B i$ on temperature field 


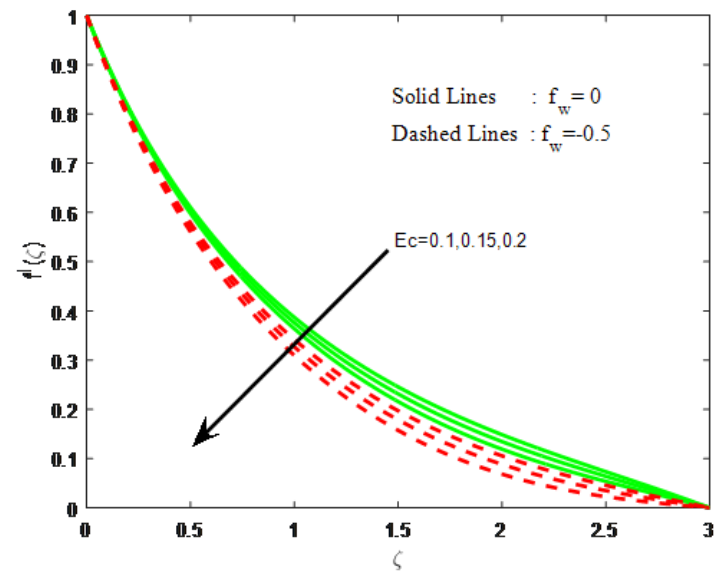

Fig. 8: The effect of Eckert number $E c$ on velocity field profile

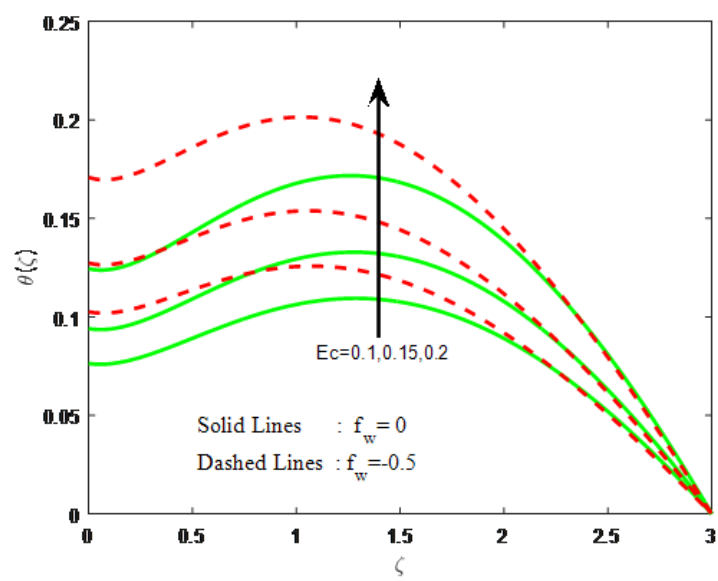

Fig. 9: The effect of Eckert number $E c$ on temperature field

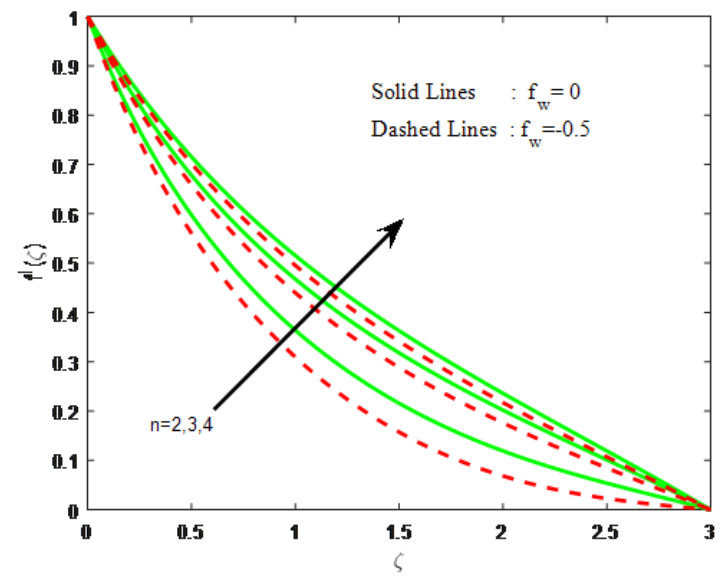

Fig. 10: The effect of power law of index $n$ on velocity field profile

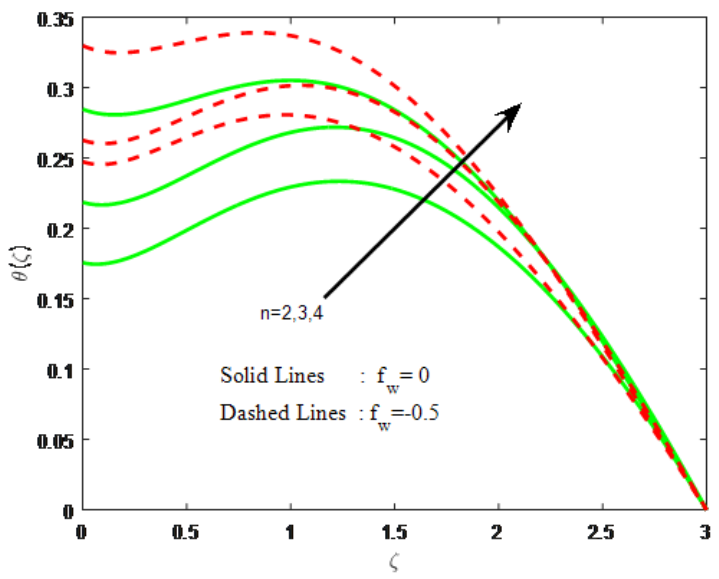

Fig. 11: The effect of power law of index $n$ on temperature field profile

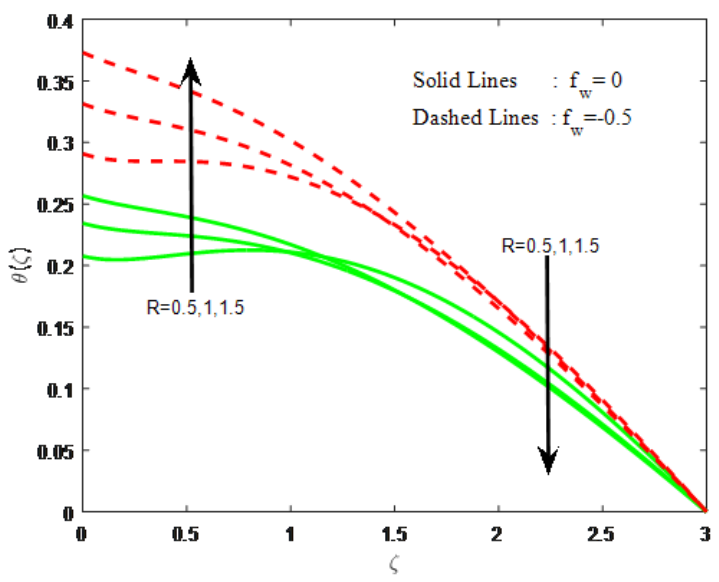

Fig. 12: The effect of Radiation parameter $R$ on temperature field profile

number $(E)$, Power law of index $(n)$, radiation parameter $(R)$, chemical reaction parameter $\left(K_{l}\right)$ and Schmidt number $(S)$ on the flow, temperature and concentration profiles. Also, the friction factor, Nusselt and Sherwood numbers are deliberated and given in tabular form for suction and injection cases separately. $M=K=0.2, G c=G r=$ $0.2, N=0.5, E c=0.2, R=0.1, S c=0.1, K_{l}=0.3, n=$ $2, Q_{H}=0.5, W e=0.2$; these valves we used for numerical results. These values are well-kept as common throughout the study except the different values in respective figures and tables. In this study the graphs in solid and dashed line indicates the impermeable $\left(f_{w}=0\right)$ and permeable $\left(f_{w} \neq 0\right)$ cases respectively.

Figs. 2 and 3 represent the effect of magnetic field parameter $(M)$ on velocity and temperature profiles. It is clear from the figures that an increase in the magnetic 


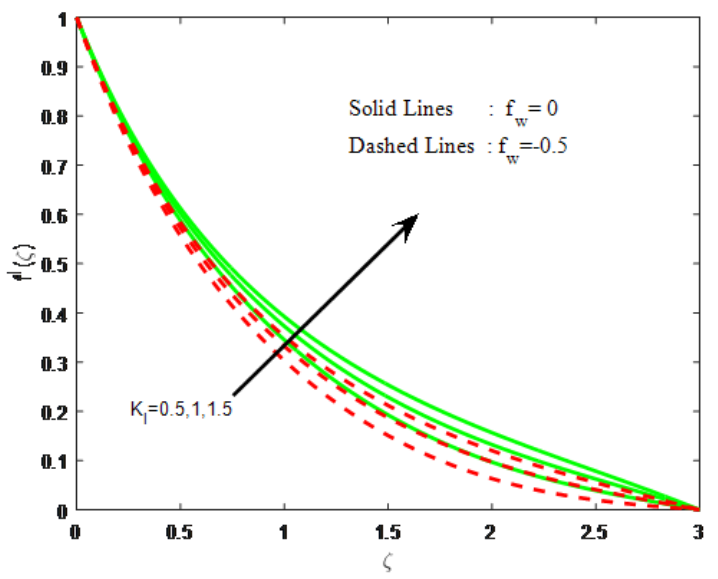

Fig. 13: The effect of chemical reaction parameter $\left(K_{l}\right)$ on velocity field

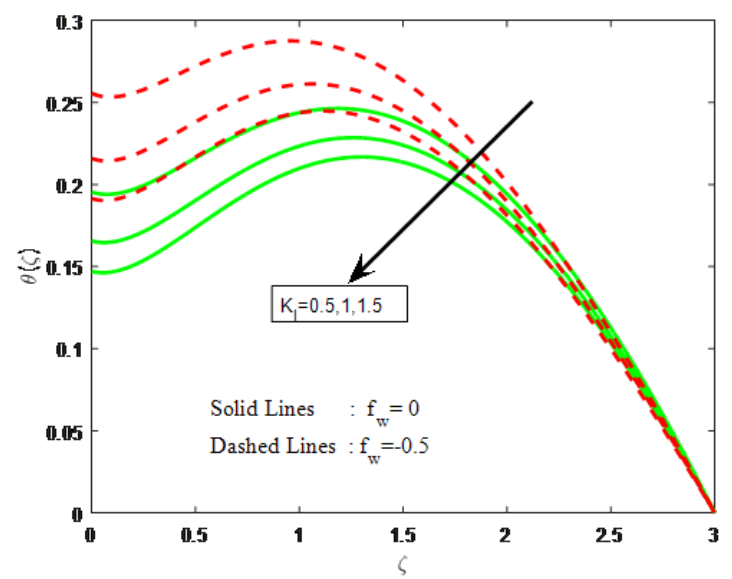

Fig. 14: The effect of chemical reaction parameter $\left(K_{l}\right)$ on temperature field

field parameter decays the velocity profile and temperature boundary layers. This is due to the fact that enhance in magnetic field develops the opposed force to the flow direction, which is called Lorentz force. This force we have has the tendency to reduce the velocity boundary layer and increase the thermal boundary layer thickness. Figs. 4 and 5 display the effect of heat source parameter $\left(Q_{H}\right)$ on velocity and temperature profiles respectively. It is evident from the figures that an increase in heat source parameter enhances the velocity profile and decreases the temperature profile. In general as improving values of heat source acts as generation and heat sink acts as a heat absorption in the flow. Figs. $6-7$ depict the Biot Number (Bi) on velocity field and temperature profiles. It is noticed that increase in Biot Number parameter increases the velocity profile and decays the temperature profile. The increas-

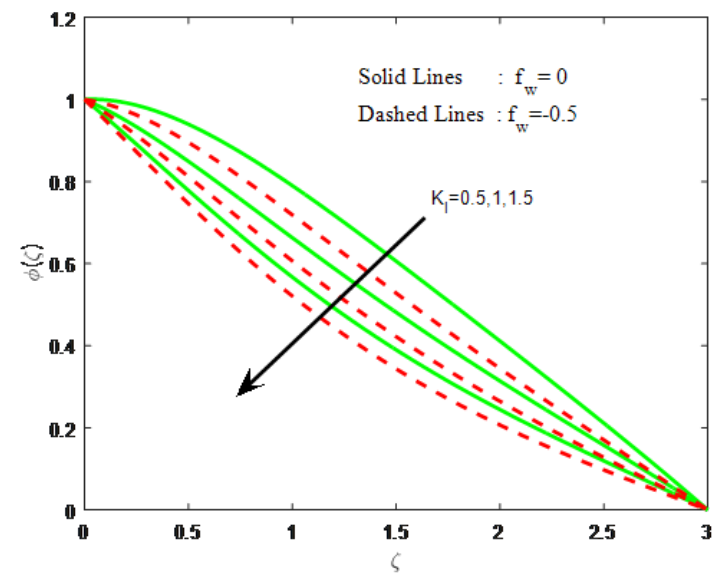

Fig. 15: The effect of chemical reaction parameter $\left(K_{l}\right)$ on concentration field

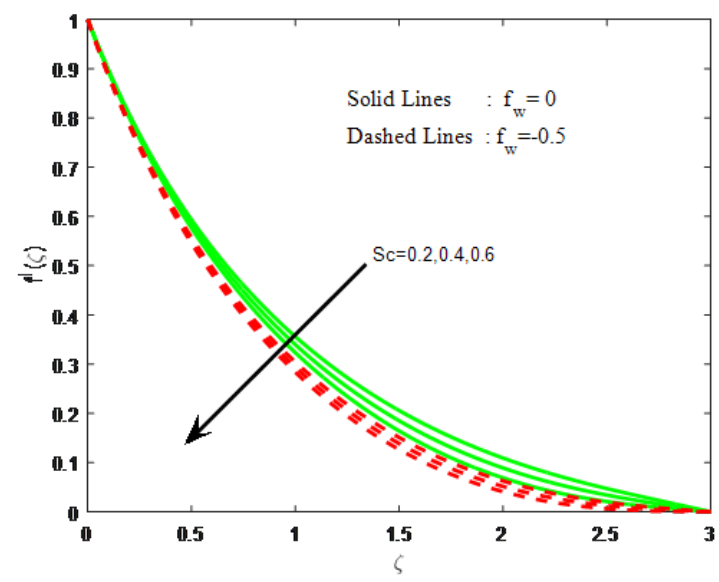

Fig. 16: The effect of Schmidt number $(S c)$ on velocity field

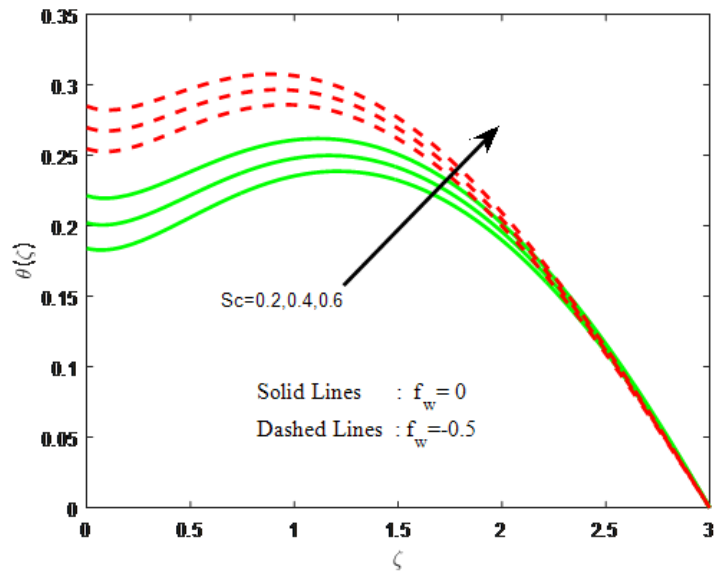

Fig. 17: The effect of Schmidt number $(S c)$ on temperature field 


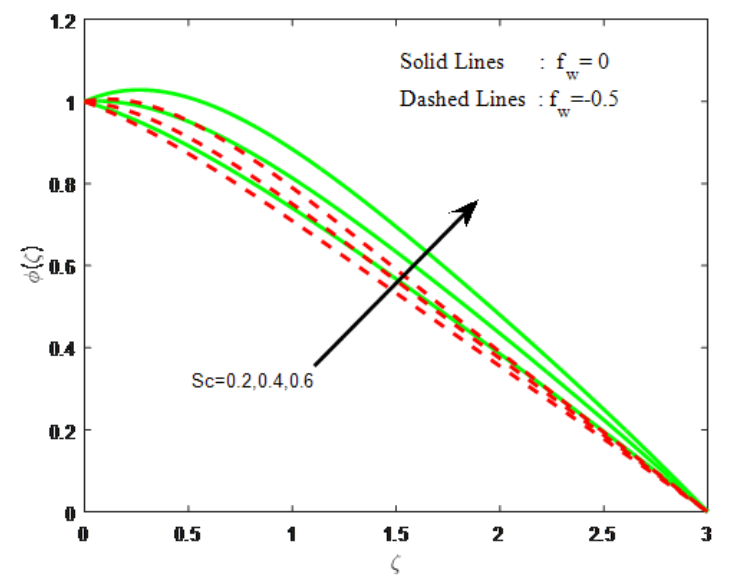

Fig. 18: The effect of Schmidt number $(S c)$ on concentration field

ing in Biot number encourages the heat transfer coefficient value; due to this we have seen enhancement in velocity and the dominance of heat transfer coefficient decrement in temperature field. Figs. 8 - 9 represent the Eckert number $(E c)$ on velocity field and temperature profiles. It is noticed that enhanced in the Eckert number declines the velocity profile and increase the temperature profile. Figs. 10 and 11 display the power law of index $(n)$ on velocity field and temperature profiles. It can be observed that there is increasing the power law of index enrichment in the velocity and temperature profiles.

The impact of thermal radiation parameter $(R)$ on temperature profile is shown in Fig. 12. It can be observed that there is enrichment in the temperature profile, whereas after certain points decline the temperature profile when there is an increasing the thermal radiation parameter. In general as increasing thermal radiation improves the heat energy in the flow due to this we saw increment in the flow. The stimulus of chemical reaction parameter $\left(K_{l}\right)$ on velocity field profile, temperature profile and concentration is presented in Figs. 13 - 15. It can be detected from the figure that an increase in the value of chemical reaction parameter enhances the velocity profile and reduces the temperature and concentration boundary layers. The rising value of chemical reaction the intermolecular interaction is more because of this we saw decrement in concentration profiles. Figs. 16 - 18 displays the Schmidt number $(S c)$ on velocity field profile, temperature profile and gas concentration field. It can be notice that increasing Schmidt number enhance the temperature and concentration whereas reduce the velocity field profile. Table 1 shows the variations of local skin friction factor, Nusselt and Sherwood numbers coefficient for various physical parameters in Suction case. Magnetic field parameter has inclination to increases the skin friction factor and decays the mass transfer rate and heat transfer rate. This happened due to Lorentz force. Heat source parameter has enhances the skin friction factor and decreases the Nusselt and Sherwood numbers. Increasing the Biot number it is observed that increases the skin friction and Nusselt number, reduces the Sherwood number. As improving Biot number improves the heat transfer coefficient value, this helps to improve the heat transfer rate. It observed that Nusselt and Sherwood numbers increases and skin friction decreases with increasing Eckert number. Increase in power law of index increases the skin friction coefficient, heat transfer rate and decline the mass transfer rate. Increasing the radiation parameter it is found to enhance the skin friction, heat transfer and mass transfer rate. Chemical reaction parameter has the tendency to reduce the heat transfer rate and increases the skin friction, Sherwood number. Increasing the Schmidt number increases the Nusselt number and reduces the skin friction factor, Sherwood number. Table 2 displays the validation of current solutions with already existing studies under limited case and we found that the results are coinciding with those solutions. Table 3 shows the variations of local skin friction factor, Nusselt and Sherwood numbers coefficient for various physical parameters in Injection case. Magnetic field parameter has inclination to increases the skin friction factor and decreases the mass transfer rate and heat transfer rate. Heat source parameter has enhances the skin friction factor and decays the Nusselt and Sherwood numbers. Increasing the Biot number it is observed that increases the skin friction and Nusselt number, reduces the Sherwood number. It observed that Nusselt and Sherwood numbers increases and skin friction decreases with increasing Eckert number. Increase in power law of index increases the heat transfer rate and decline the skin friction coefficient, mass transfer rate. Increasing the radiation parameter it is found to decreases the skin friction and increases the heat transfer and mass transfer rate. Chemical reaction parameter has the tendency to reduce the heat transfer rate and increases the skin friction, Sherwood number. Increasing the Schmidt number increases the heat transfer rate and reduces the skin friction, mass transfer rate.

\section{Conclusions}

This study presented the steady-state magnetohydrodynamic boundary layer flow behavior of tangent hyperbolic fluid over an exponentially stretching surface in the existence of heat source and chemical reaction. The partial 
Table 1: The variations of local skin friction, Nusselt and Sherwood numbers for various values of $M=0.2, K=0.2, G c=G r=0.2, N=$ $0.5, E c=0.2, R=0.1, S c=0.1, K_{l}=0.3, n=2, Q_{H}=0.5, W e=0.2$ in $f_{w}=0$ case.

\begin{tabular}{|c|c|c|c|c|c|c|c|c|c|c|}
\hline$M$ & $Q_{H}$ & $B i$ & $E c$ & $n$ & $R$ & $K_{l}$ & $S c$ & Skin & Nus & Shr \\
\hline 0.2 & & & & & & & & -1.412995 & 0.001551 & 0.234896 \\
\hline 0.4 & & & & & & & & -1.383840 & 0.001261 & 0.228382 \\
\hline 0.6 & & & & & & & & -1.352382 & 0.001002 & 0.220939 \\
\hline & 0.2 & & & & & & & -1.438090 & 0.060166 & 0.242807 \\
\hline & 0.4 & & & & & & & -1.433932 & 0.045759 & 0.241927 \\
\hline & 0.6 & & & & & & & -1.430956 & 0.034435 & 0.241402 \\
\hline & & 0.1 & & & & & & -1.433718 & 0.020632 & 0.242167 \\
\hline & & 0.15 & & & & & & -1.433009 & 0.030393 & 0.241892 \\
\hline & & 0.2 & & & & & & -1.432331 & 0.039827 & 0.241628 \\
\hline & & & 0.1 & & & & & -1.421200 & 0.017308 & 0.237800 \\
\hline & & & 0.15 & & & & & -1.426445 & 0.027767 & 0.239619 \\
\hline & & & 0.2 & & & & & -1.432331 & 0.039827 & 0.241628 \\
\hline & & & & 2 & & & & -1.432331 & 0.039827 & 0.241628 \\
\hline & & & & 3 & & & & -2.487293 & 0.049547 & 0.223369 \\
\hline & & & & 4 & & & & -3.553227 & 0.064548 & 0.214905 \\
\hline & & & & & 0.5 & & & -1.432157 & 0.069246 & 0.240876 \\
\hline & & & & & 1 & & & -1.433005 & 0.109399 & 0.240788 \\
\hline & & & & & 1.5 & & & -1.434032 & 0.154079 & 0.240886 \\
\hline & & & & & & 0.5 & & -1.440641 & 0.044297 & -0.027134 \\
\hline & & & & & & 1 & & -1.429160 & 0.037545 & 0.215321 \\
\hline & & & & & & 1.5 & & -1.421012 & 0.033335 & 0.415197 \\
\hline & & & & & & & 0.2 & -1.435747 & 0.041730 & 0.148681 \\
\hline & & & & & & & 0.4 & -1.442763 & 0.045823 & -0.039368 \\
\hline & & & & & & & 0.6 & -1.449841 & 0.050189 & -0.227955 \\
\hline
\end{tabular}

Table 2: Comparison present solutions with already available results with limited case.

\begin{tabular}{lllll}
\hline & Pranmik [27] & Ishak [28] & Raju et al. [13] & Present results \\
\hline 1 & 0.9547 & 0.9548 & 0.9547 & 0.95472 \\
2 & 1.4714 & 1.4715 & 1.475 & 1.47492 \\
3 & 1.8691 & 1.8691 & 1.8691 & 1.86921 \\
10 & 3.6603 & 3.6603 & 3.6603 & 3.66032 \\
\hline
\end{tabular}

nonlinear differential equations are transformed into ordinary differential equations by using the similarity conversion and the accomplished boundary layer ordinary differential equations are elucidated numerically by using Matlab bvp4c package. The effects of numerous nondimensional governing factors on velocity, temperature and gas concentration profiles are deliberated and presented graphically. Also, the Skin friction factor, Nusselt and Sherwood numbers are analyzed and presented in tabular form for suction and injection cases separately.

- Magnetic field parameter has inclination to decays the heat and mass transfer rate.

- Heat source parameter enhances the skin friction factor and reduces the heat transfer, mass transfer rate.

- Biot number enhances the skin friction, Nusselt number and decrease the Sherwood number.
- Chemical reaction parameter has the tendency to reduce the heat transfer rate.

- An increase in power law of index the increases the heat transfer rate and decline the mass transfer rate.

- It observed that Nusselt and Sherwood numbers increases and skin friction decreases with increasing Eckert number.

\section{Nomenclature}

$u, v$ : Velocity of components in $x$ and $y$ directions respectively

$x$ : Direction along the surface

$y$ : Direction normal to the surface

$S$ : Extra stress tensor

$n$ : Power law index

$\overline{y_{i j}}$ : Second invariant strain tensor 
Table 3: The variations of local skin friction, Nusselt and Sherwood numbers for several of $M=0.2, K=0.2, G c=G r=0.2, N=0.5, E c=$ $0.2, R=0.1, S c=0.1, K_{l}=0.3, n=2, Q_{H}=0.5, W e=0.2$ in $f_{w}=-0.5$ case.

\begin{tabular}{|c|c|c|c|c|c|c|c|c|c|c|}
\hline$M$ & $Q_{H}$ & $B i$ & $E c$ & $n$ & $R$ & $K_{l}$ & Sc & Skin & Nus & Shr \\
\hline 0.2 & & & & & & & & -1.447207 & 0.002029 & 0.254137 \\
\hline 0.4 & & & & & & & & -1.417374 & 0.001661 & 0.247830 \\
\hline 0.6 & & & & & & & & -1.384822 & 0.001324 & 0.240495 \\
\hline & 0.2 & & & & & & & -1.486626 & 0.090767 & 0.265581 \\
\hline & 0.4 & & & & & & & -1.478288 & 0.065449 & 0.263684 \\
\hline & 0.6 & & & & & & & -1.472998 & 0.048020 & 0.262603 \\
\hline & & 0.1 & & & & & & -1.477913 & 0.029619 & 0.263929 \\
\hline & & 0.15 & & & & & & -1.476603 & 0.043197 & 0.263486 \\
\hline & & 0.2 & & & & & & -1.475383 & 0.056091 & 0.263071 \\
\hline & & & 0.1 & & & & & -1.458647 & 0.023248 & 0.257829 \\
\hline & & & 0.15 & & & & & -1.466321 & 0.038066 & 0.260255 \\
\hline & & & 0.2 & & & & & -1.475383 & 0.056091 & 0.263071 \\
\hline & & & & 2 & & & & -1.475383 & 0.056091 & 0.263071 \\
\hline & & & & 3 & & & & -2.519950 & 0.059567 & 0.240499 \\
\hline & & & & 4 & & & & -3.583283 & 0.074787 & 0.230709 \\
\hline & & & & & 0.5 & & & -1.476023 & 0.096971 & 0.262611 \\
\hline & & & & & 1 & & & -1.478020 & 0.154709 & 0.262840 \\
\hline & & & & & 1.5 & & & -1.480947 & 0.223865 & 0.263483 \\
\hline & & & & & & 0.5 & & -1.479122 & 0.057911 & 0.071335 \\
\hline & & & & & & 1 & & -1.466129 & 0.048928 & 0.303968 \\
\hline & & & & & & 1.5 & & -1.456986 & 0.043388 & 0.497797 \\
\hline & & & & & & & 0.2 & -1.477941 & 0.057759 & 0.191542 \\
\hline & & & & & & & 0.4 & -1.483054 & 0.061158 & 0.044987 \\
\hline & & & & & & & 0.6 & -1.488109 & 0.064589 & -0.105846 \\
\hline
\end{tabular}

$C_{p}$ : Heat capacitance of fluid

$f$ : Dimensionless velocity for fluid Phase

$N$ : Exponential parameter

$T$ : Temperature of the fluid

$K_{l}$ : Chemical reaction parameter

$k$ : Thermal conductivity

We: Weissennberg number

g: Acceleration due to gravity

$q_{r}$ : Relative heat flux

$\beta_{T}$ : Thermal expansion coefficient

$\beta_{c}$ : Concentration expansion coefficient

$\tau$ : Time dependent constant

$\theta$ : Temperature of fluid phase

$D_{m}$ : Coefficient of mass diffusivity

$V(x)$ : Suction/injection parameter

$U_{0}$ : Fluid velocity

$U$ : Constant velocity

Ec: Eckert number

$R$ : Radiation parameter

Sc: Schmidt number

Gr: Thermal Grashof number

$G c$ : Concentration Grashof number

$C$ : Concentration of the fluid

$T$ : Fluid temperature

$\rho$ : Density of the fluid $\mu_{0}$ : Zero shear rate viscosity

$\mu_{\infty}$ : Infinite shear rate viscosity

$Q_{0}$ : Variable heat source parameter

$T_{\infty}$ : Ambient fluid temperature

$C_{\infty}$ : Ambient fluid concentration Pr: Prandtl number

$Q_{H}$ : Heat source parameter

$B$ : Variable magnetic field parameter

$M$ : Magnetic field parameter

$B i$ : Biot number

$C_{f}$ : Skin friction coefficient

$N u_{x}$ : Local Nusselt number

$R e_{x}$ : Local Reynolds number

\section{Greek Symbols}

$\phi$ : Dimensionless concentration

$\beta$ : Local fluid interaction parameter

$\xi$ : Similarity variable

$\sigma$ : Electrical conductivity of the fluid

$y$ : Ratio of specific heats

$\theta$ : Dimensionless temperature

$\rho$ : Density of the fluid

$\mu$ : Dynamic viscosity

$v$ : Kinematic viscosity 


\section{References}

[1] S. Nadeem, S. Akram, Magnetohydrodynamic peristaltic flow of a hyperbolic tangent fluid in a vertical asymmetric channel with heat transfer, Acta Mech. Sin. Xuebao. 27 (2011) 237-250. doi:10.1007/s10409-011-0423-2.

[2] S.A. Gaffar, V.R. Prasad, O.A. Bég, Numerical study of flow and heat transfer of non-Newtonian Tangent Hyperbolic fluid from a sphere with Biot number effects, Alexandria Eng. J. 54 (2015) 829-841. doi:10.1016/j.aej.2015.07.001.

[3] M. Gnaneswara Reddy, J. Manjula, P. Padma, Mass Transfer Flow of MHD Radiative Tangent Hyperbolic Fluid over a Cylinder: A Numerical Study, Int. J. Appl. Comput. Math. 3 (2017) 447-472. doi:10.1007/s40819-017-0364-y.

[4] H. Xu, S.J. Liao, Laminar flow and heat transfer in the boundary-layer of non-Newtonian fluids over a stretching flat sheet, Comput. Math. with Appl. 57 (2009) 1425-1431. doi:10.1016/j.camwa.2009.01.029.

[5] A.M. Megahed, Flow and heat transfer of a non-Newtonian power-law fluid over a non-linearly stretching vertical surface with heat flux and thermal radiation, Meccanica. 50 (2015) 1693-1700. doi:10.1007/s11012-015-0114-3.

[6] M. Gnaneswara Reddy, P. Padma, B. Shankar, Effects of viscous dissipation and heat source on unsteady MHD flow over a stretching sheet, Ain Shams Eng. J. 6 (2015) 1195-1201. doi:10.1016/j.asej.2015.04.006.

[7] M.K. Choudhary, S. Chaudhary, R. Sharma, Unsteady MHD Flow and Heat Transfer over a Stretching Permeable Surface with Suction or Injection, Procedia Eng. 127 (2015) 703-710. doi:10.1016/j.proeng.2015.11.371.

[8] A. Hussain, M.Y. Malik, T. Salahuddin, A. Rubab, M. Khan, Effects of viscous dissipation on MHD tangent hyperbolic fluid over a nonlinear stretching sheet with convective boundary conditions, Results Phys. 7 (2017) 3502-3509. doi:10.1016/j.rinp.2017.08.026.

[9] M.K. Partha, P.V.S.N. Murthy, G.P. Rajasekhar, Effect of viscous dissipation on the mixed convection heat transfer from an exponentially stretching surface, Heat Mass Transf. Und Stoffuebertragung. 41 (2005) 360-366. doi:10.1007/s00231004-0552-2.

[10] K. Uma, G. Koteswara, Boundary Layer Flow Of MHD Tangent Hyperbolic Fluid Past A Vertical Plate In The Presence Of Thermal Dispersion Using Spectral Relaxation Method, (2017) 2841.

[11] S.U. Mamatha, Mahesha, C.S.K. Raju, O.D. Makinde, Effect of convective boundary conditions on MHD carreau dusty fluid over a stretching sheet with heat source, Defect Diffus. Forum. 377 (2017). doi:10.4028/www.scientific.net/DDF.377.233.

[12] S.A. Gaffar, V.R. Prasad, S.K. Reddy, O.A. Bég, Magnetohydrodynamic free convection boundary layer flow of nonNewtonian tangent hyperbolic fluid from a vertical permeable cone with variable surface temperature, J. Brazilian Soc. Mech. Sci. Eng. 39 (2017) 101-116. doi:10.1007/s40430-016-0611-x.

[13] C.S.K. Raju, N. Sandeep, V. Sugunamma, M. Jayachandra Babu, J. V. Ramana Reddy, Heat and mass transfer in magnetohydrodynamic Casson fluid over an exponentially permeable stretching surface, Eng. Sci. Technol. an Int. J. 19 (2016) 4552. doi:10.1016/j.jestch.2015.05.010.
[14] H. Dessie, N. Kishan, MHD effects on heat transfer over stretching sheet embedded in porous medium with variable viscosity, viscous dissipation and heat source/sink, Ain Shams Eng. J. 5 (2014) 967-977. doi:10.1016/j.asej.2014.03.008.

[15] M. Naseer, M.Y. Malik, S. Nadeem, A. Rehman, The boundary layer flow of hyperbolic tangent fluid over a vertical exponentially stretching cylinder, Alexandria Eng. J. 53 (2014) 747-750. doi:10.1016/j.aej.2014.05.001.

[16] M.S. Abel, P.S. Datti, N. Mahesha, Flow and heat transfer in a power-law fluid over a stretching sheet with variable thermal conductivity and non-uniform heat source, Int. J. Heat Mass Transf. 52 (2009) 2902-2913. doi:10.1016/j.ijheatmasstransfer.2008.08.042.

[17] S. Jyothi, M.V.S. Reddy, P. Gangavathi, Hyperbolic Tangent Fluid Flow Through A Porous Medium in an Inclined Channel with Peristalsis, 1 (2016) 113-121.

[18] G.S. K. Vendabai, Unsteady Convective Boundary Layer Flow Of A Nano Fluid Over A Stretching Surface In The Presence Of A Magnetic Field And Heat Generation, Int. J. Emerg. Trends Eng. Dev. 3 (2014) 214-230. doi:10.1017/CB09781107415324.004.

[19] S. Mansur, A. Ishak, Unsteady boundary layer flow of a nanofluid over a stretching/shrinking sheet with a convective boundary condition, J. Egypt. Math. Soc. 24 (2016) 650-655. doi:10.1016/j.joems.2015.11.004.

[20] G. Sarojamma, K. Vendabai, Boundary Layer Flow of a Casson Nanofluid past a Vertical Exponentially Stretching Cylinder in the Presence of a Transverse Magnetic Field with Internal Heat Generation / Absorption, Int. J. Mech. Aerospace, Ind. Mechatronics Eng. 9 (2015) 138-143.

[21] P. Loganathan, p. Nirmal chand, p. Ganesan, Radiation Effects on an Unsteady Natural Convective Flow of a Nanofluid Past an Infinite Vertical Plate, NANO Br. Reports Rev. 8 (2013) 1350001, 1-10. doi:10.1142/S179329201350001X.

[22] K. Ganesh Kumar, B.J. Gireesha, M.R. Krishanamurthy, N.G. Rudraswamy, An unsteady squeezed flow of a tangent hyperbolic fluid over a sensor surface in the presence of variable thermal conductivity, Results Phys. 7 (2017) 3031-3036. doi:10.1016/j.rinp.2017.08.021.

[23] T. Salahuddin, M.Y. Malik, A. Hussain, M. Awais, I. Khan, M. Khan, Analysis of tangent hyperbolic Nano fluid impinging on a stretching cylinder near the stagnation point, Results Phys. 7 (2017) 426-434. doi:10.1016/j.rinp.2016.12.033.

[24] H. C. Brinkman, Heat effects in capillary flow I, Applied Scientific Research, 2(1951)120-124.

[25] V. Ramachandra Prasad, S. Abdul Gaffar, O. Anwar Beg, Free convection flow and heat transfer of Tangent Hyperbolic past a vertical porous plate with partial slip, J. Appl. Fluid Mech. 9 (2016) 1667-1678. doi:10.18869/acadpub.jafm.68.235.24718.

[26] A.M. Rashad, A.J. Chamkha, S.M.M. El-Kabeir, Effect of chemical reaction on heat and mass transfer by mixed convection flow about a sphere in a saturated porous media, Int. J. Numer. Methods Heat Fluid Flow. 21 (2011) 418-433. doi:10.1108/09615531111123092.

[27] C. S. K. Raju, N.Sandeep, \& M. G. Reddy, Effect of nonlinear thermal radiation on 3D Jeffrey fluid flow in the presence of homogeneous-heterogeneous reactions. International Journal of Engineering Research in Africa, 21 (2015) 52.

[28] D Gopal, N Kishan, CSK Raju, Viscous and Joule's dissipation on Casson fluid over a chemically reacting stretching sheet 
with inclined magnetic field and multiple slips, Informatics in Medicine Unlocked, 9 (2017) 154-160.

[29] A.M. Rashad, S. Abbasbandy, A.J. Chamkha, Mixed convection flow of a micropolar fluid over a continuously moving vertical surface immersed in a thermally and solutally stratified medium with chemical reaction, J. Taiwan Inst. Chem. Eng. 45 (2014) 2163-2169. doi:10.1016/j.jtice.2014.07.002.

[30] A.J. Chamkha, A.M. Rashad, Unsteady heat and mass transfer by MHD mixed convection flow from a rotating vertical cone with chemical reaction and Soret and Dufour effects, Can. J. Chem. Eng. 92 (2014) 758-767. doi:10.1002/cjce.21894.

[31] P.D.Prasad, Raju, C.S.K., S.V.K. Varma, S. A. Shehzad, A. G. Madaki, Cross diffusion and multiple slips on MHD Carreau fluid in a suspension of microorganisms over a variable thickness sheet, J Braz. Soc. Mech. Sci. Eng. 40 (2018) 256. https://doi.org/10.1007/s40430-018-1171-z
[32] A.J. Chamkha, S.M.M. El-Kabeir, Unsteady Heat and Mass Transfer By Mhd Mixed Convection Flow Over an Impulsively Stretched Vertical Surface With Chemical Reaction and Soret and Dufour Effects, Chem. Eng. Commun. 200 (2013) 12201236. doi:10.1080/00986445.2012.743893.

[33] Nagendramma, V., Leelarathnam, A., Raju, C. S. K., Shehzad, S. A., \& Hussain, T. (2018). Doubly stratified MHD tangent hyperbolic nanofluid flow due to permeable stretched cylinder. Results in Physics, 9, 23-32.

[34] A.M. Rashad, A. Chamkha, MHD Free Convective Heat and Mass Transfer of a Chemically-Reacting Fluid from Radiate Stretching Surface Embedded in a Saturated Porous Medium MHD Free Convective Heat and Mass Transfer of a ChemicallyReacting Fluid, 9 (2011). doi:10.1515/1542-6580.2501. 